\title{
Downregulation of BMP6 enhances cell proliferation and chemoresistance via activation of the ERK signaling pathway in breast cancer
}

\author{
WEN-JING LIAN ${ }^{1}$, GENG LIU ${ }^{1}$, YUAN-JIE LIU ${ }^{1}$, ZHI-WEI ZHAO ${ }^{1}$, TAO YI ${ }^{2}$ and HONG-YING ZHOU ${ }^{1}$ \\ ${ }^{1}$ Department of Human Anatomy, West China School of Preclinical and Forensic Medicine, Sichuan University, \\ Chengdu, Sichuan 610041; ${ }^{2}$ Biotherapy Laboratory of Gynecological Oncology, Key Laboratory of Obstetric \\ and Gynecologic and Pediatric Diseases and Birth Defects of the Ministry of Education, \\ West China Second Hospital, Sichuan University, Chengdu, Sichuan 610041, P.R. China
}

Received January 16, 2013; Accepted April 8, 2013

DOI: $10.3892 /$ or.2013.2462

\begin{abstract}
Previous studies indicate that bone morphogenetic protein (BMP) 6 is involved in breast cancer development and progression. However, the mechanism underlying the role of BMP6 in breast cancer cell proliferation, differentiation and chemoresistance remains unknown. In this study, we confirmed that BMP6 expression was downregulated in breast cancer tissues compared with the adjacent normal breast tissues. We further demonstrated that the downregulation of BMP6 was correlated with the estrogen receptor (ER) and progesterone receptor (PR) status, tumor grade and enhanced proliferation (Ki67 proliferation index). In vitro functional experiments showed that the suppression of BMP6 expression by a specific small hairpin (sh)RNA vector led to increased proliferation in the MCF7 breast cancer cell line. Furthermore, knockdown of BMP6 in MCF7 cells enhanced the chemoresistance to doxorubicin by upregulation of mdr-1/P-gp expression and activation of the ERK signaling pathway. Taken together, our data suggest that BMP6 plays a critical role in breast cancer cell aberrant proliferation and chemoresistance and may serve as a novel diagnostic biomarker or therapeutic target for breast cancer.
\end{abstract}

Correspondence to: Dr Hong-Ying Zhou, Department of Human Anatomy, West China School of Preclinical and Forensic Medicine, Sichuan University, Renmin South Road No. 17, Chengdu, Sichuan 610041, P.R. China

E-mail: eaglezhyxzy@163.com

Dr Tao Yi, Biotherapy Laboratory of Gynecological Oncology, Key Laboratory of Obstetric and Gynecologic and Pediatric Diseases and Birth Defects of the Ministry of Education, West China Second Hospital, Sichuan University, Chengdu, Sichuan 610041, P.R. China

E-mail: yitao-yt@sohu.com

Key words: bone morphogenetic protein 6, breast cancer, chemoresistance, ERK signaling pathway

\section{Introduction}

Breast cancer is one of the leading causes of cancer-related mortality among women worldwide. Recent studies have shown that an increasing number of multiple signaling pathways and genes are involved in breast cancer tumorigenesis (1-3). However, the precise mechanisms determining breast cancer cell aberrant proliferation, differentiation and sensitivity to chemotherapy remain unknown. Bone morphogenetic proteins (BMPs), belonging to the transforming growth factor (TGF)- $\beta$ superfamily, have been widely considered as crucial molecules involved in cell proliferation, differentiation, apoptosis and migration in various tissues $(4,5)$. Currently, they are well known to play critical roles in tumorigenesis and progression of breast cancer, prostate cancer, multiple myeloma, and ovarian cancer (6). BMP6 has been detected in various types of cancer and cell lines, and is associated with cancer cell growth, migration and drug resistance (7). In breast cancer, BMP6 was found to function as an inhibitor of cancer cell growth and migration (7). A recent study found that the BMP inhibitor Coco was also able to reactivate breast cancer cells at lung metastatic sites (2). These findings indicate that BMP6 is involved in breast cancer development and progression. However, the mechanism underlying the role of BMP6 in breast cancer cell proliferation, differentiation and chemoresistance remains unclear. Therefore, a further understanding of the molecular mechanism of BMP6 in breast cancer is urgently needed.

In the present study, we first investigated the expression pattern of BMP6 to determine its potential role in breast cancer tumorigenesis. We showed that BMP6 was downregulated in breast cancer and was closely related to cancer cell proliferation and tumor grade. Therefore, we evaluated the function of BMP6 in proliferation and the chemoresistance of breast cancer cells. Our study revealed that suppression of BMP6 expression in breast cancer cell line MCF7 increased cell proliferation and chemoresistance via activation of the ERK signaling pathway. Based on the data from these experiments, BMP6 plays an important role in breast cancer and may serve as a diagnostic biomarker and potential therapeutic target for breast cancer. 


\section{Materials and methods}

Patients and tissue specimens. Thirty-six fresh breast cancer tissues and patient-matched adjacent normal breast tissues were obtained from patients who underwent surgery at West China Hospital of Sichuan University. All tumor tissues were reviewed by an experienced pathologist using World Health Organization recommendations on histopathologic typing. Parts of the fresh specimens were frozen in liquid nitrogen and stored at $-80^{\circ} \mathrm{C}$ for total RNA and protein isolation. Other parts were fixed in $4 \%$ paraformaldehyde and embedded in paraffin for histological sections and immunohistochemical staining. In addition, 80 paraffin-embedded breast cancer tissues were obtained from the Cancer Hospital of Sichuan Province for immunohistochemical staining. None of the patients received chemotherapy or radiotherapy prior to surgery. The study protocol conformed to the local ethical standards of the institutional review board.

Immunohistochemistry. Immunostaining was performed on paraffin-embedded sections. The sections were dewaxed in xylene and immersed in graded ethanol and distilled water. Immunohistochemical staining was performed using the avidin-biotin peroxidase complex (ABC) method, according to the manufacturer's instructions. The primary antibody for BMP6 (Abcam) was diluted to 1:200. The primary antibody was omitted as a negative control for the immunostaining. The tissue specimens were viewed separately by two pathologists under double-blind conditions. The immunoreactivity score system was in accordance with a previously described system (8).

RNA isolation and quantitative RT-PCR. Total RNA from 36 cancer and adjacent normal breast tissues was extracted using TRIzol (Invitrogen) according to the manufacturer's instructions. Total RNA was retrotranscribed using the RevertAid $^{\mathrm{TM}}$ First Strand cDNA Synthesis kit (Fermentas) according to the manufacturer's instructions. PCR amplification and detection of the PCR amplified gene products were performed with SYBR-Green PCR Master Mix (Tiangen). Real time-PCR was performed on Mastercycler ${ }^{\circledR}$ ep realplex (Eppendorf). The reaction cycle consisted of a hot start at $95^{\circ} \mathrm{C}$ for $10 \mathrm{~min}$, then 30 cycles of denaturation at $95^{\circ} \mathrm{C}$ for $30 \mathrm{sec}$ and extension at $65^{\circ} \mathrm{C}$ for $30 \mathrm{sec}$. Levels of mRNA expression were quantified after normalization with endogenous control GAPDH using the $2^{-\Delta C T}$ method (9). The primer sequences used for PCR were as follows: GAPDH, 5'-ctttggtatcgtggaaggactc-3' and 5'-gtagaggcagggatgatgttct-3' [product size, 132 base pairs (bp)]; bmp6, 5'-gtcttacaggagcatcagcacag-3' and 5'-ggagtcacaacccacagattg-3' (product size, 128 bp); mdr-1, 5 '-gctcctgactatgccaaagc-3' and 5'-tcttcacctccaggctcagt-3' (product size, $202 \mathrm{bp}$ ). Experiments were performed independently for each sample and at least 3 technical replicates were run for each treated sample and controls.

Restriction fragment differential display (RFDD)-PCR. Total-RNA of the cancer and adjacent normal breast tissues was extracted respectively by TRIzol according to the manufacturer's instructions. RFDD-PCR for each sample was accomplished by Display PROfile ${ }^{\mathrm{TM}}$ kits (cat. nos. 600-100, 615-100, 616-100, 617-100, 618-100) provided by Qbiogene,
Inc. and MP Biomedicals, Inc. (Carlsbad, CA, USA) as previously described and used in our laboratory $(10,11)$. After RFDD-PCR, $15 \mu 1$ loading buffer was added, and the samples were heated to $85^{\circ} \mathrm{C}$ for $5 \mathrm{~min}$ and placed directly on ice. Each sample (5 $\mu \mathrm{l})$ was loaded on a $7 \%$ urea polyacrylamide gel that had been pre-run for $30 \mathrm{~min}$ at $60 \mathrm{~W}$ in a high-voltage vertical electrophoresis system (Bio-Rad, Hercules, CA, USA) and 0.6X Tris-borate (TBE)-ethylenediaminetetraacetic acid (EDTA) was used as the electrophoresis buffer. Gels were scanned with Typhoon 9200 variable mode imager (GE Healthcare Biosciences). The scanning parameters consisted of a wavelength of 33/670 nm, PMT voltage of $625 \mathrm{~V}$ and scanning precision of $200 \mu \mathrm{m}$. Based on the linear correlation between the molecule weight (bp) of DNA and its space of shift in gel, the standard curve was obtained with UTHSCSA Image Tool and Excel 2000 for each gel resulting in acquisition of the size (bp) of each fragment of DNA. According to the number of $\mathrm{bp}$, genes were determined by searching in the corresponding database provided by Qbiogene, Inc. and MP Biomedicals, Inc. (http://www.qbio-gene.com/displayft/).

Cell culture. The MCF7 cell line was purchase from American Type Culture Collection (ATCC), and doxorubicin resistant subline (MCF7/Adr) was generated by continuously culturing the drug-sensitive parental cell line (MCF7) in medium containing incrementally increasing concentrations of doxorubicin (Sigma-Aldrich). The cells were cultured in RPMI-1640 (Gibco) supplemented with $10 \%$ heat inactivated fetal calf serum, $100 \mathrm{U} / \mathrm{ml}$ penicillin and $100 \mathrm{U} / \mathrm{ml}$ streptomycin at $37^{\circ} \mathrm{C}$ and $5 \% \mathrm{CO}_{2}$. The drug resistant cell line was cultured in drug-free medium for over 2 weeks before subsequent experiments to avoid the influence of the drug.

BMP6 small hairpin (sh)RNA transfection and isolation of clones stably expressing BMP6 shRNA. BMP6-specific shRNA (pGPU6/GFP/NEO-BMP6) and a control shRNA vector (pGPU6/GFP/NEO-shNC) were purchased from Genepharma (Shanghai, China). Aiming at the sequence of BMP6, two DNA chains with the following sense and antisense sequences were synthesized: shRNA-1, 5'-GCT AAATGCCATCTCGGTTCT-3' and shRNA-2, 5'-GGT TGTGACTCCACAGCATAA-3'. The target sequence of the negative control group which was named pGPU6/GFP/ NEO-shNC was 5'-GTTCTCCGAACGTGTCAC GT-3', which has no homology with that of human beings or mice. MCF7 cells grown on 24-well plates were transfected either with pGPU6/GFP/NEO-BMP6 or the negative control plasmid. Transfection was performed using Lipofectamine 2000. The cells were incubated at $37^{\circ} \mathrm{C}$ in $5 \% \mathrm{CO}_{2}$ overnight, and then DMEM plus $10 \%$ FCS was added. After another 12-h incubation, the cells were re-plated at a 1/10-1/40 dilution onto 6-well plates. Selection with $\mathrm{G} 418(500 \mu \mathrm{g} / \mathrm{ml})$ was initiated on the next day and the process lasted for 2 weeks. MCF7 cell clones with stably decreased expression of BMP6 as well as the control clones were obtained for further study. BMP6 knockdown was evaluated by real-time PCR and western blotting with antibodies to BMP6.

Cell proliferation assay. Proliferation of cells stably transfected with shRNAs for BMP6 or the negative control vector was 


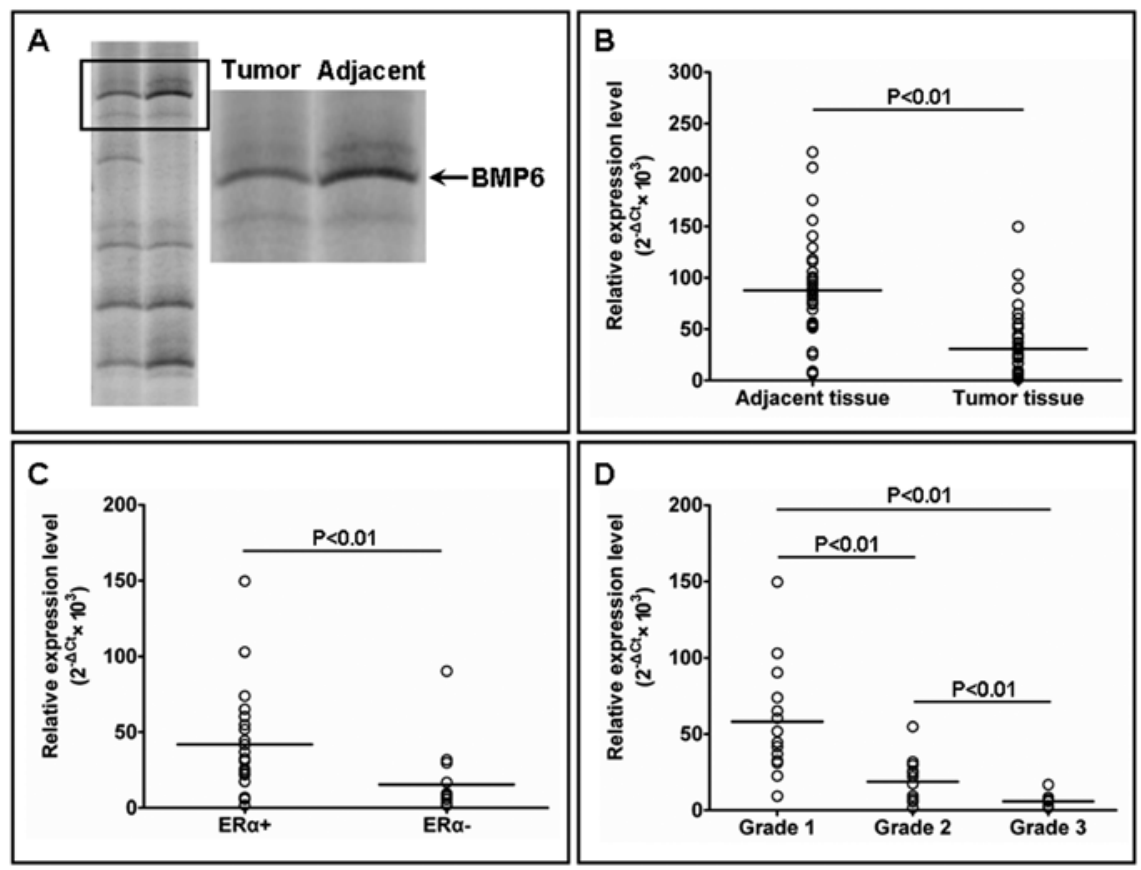

Figure 1. RFDD-PCR and real-time PCR analyese of the BMP6 expression in breast cancer and adjacent normal tissues. (A) RFDD-PCR analysis showed that the differentially expressed gene fragment representing BMP6 was downregulated in the tumor tissues. (B) Differential expression of BMP6 between breast cancer and adjacent normal tissues by RT-PCR $(n=36)$. (C) BMP6 was expressed at a much higher level in ER $\alpha$-positive breast cancer tissues than that in the ER $\alpha$-negative tissues. (D) BMP6 expression was significantly decreased in grade 2 and 3 tumors when compared with grade 1.

determined with the Cell Counting Kit-8 (CCK-8) assay and BrdU labeling. Briefly, cells of each group (1,000 cells/well) were plated in 96-well plates. After culturing for 1-5 days, the supernatant was removed and cell growth was detected using the CCK-8 Kit (Dojindo Laboratories, Kumamoto, Japan) according to the manufacturer's instructions. Absorbance was measured at $450 \mathrm{~nm}$ using a microplate reader (Thermo Fisher Scientific). All experiments were performed in triplicate and repeated at least three times. As for BrdU labeling, the cells were treated with BrdU for $2 \mathrm{~h}$. Cells were fixed and acidtreated, followed by mouse anti-BrdU antibody (diluted 1:200; Millipore) incubation for $2 \mathrm{~h}$ at $37^{\circ} \mathrm{C}$. The cells were then immunostained with FITC-conjugated secondary antibody. Quantitative studies were based on four or more replicates.

Growth inhibition assay. Cells from each group were seeded at $4 \times 10^{3}$ cells/well in 96-well tissue culture plates. After $24 \mathrm{~h}$, cells were exposed to increasing concentrations of doxorubicin (Sigma-Aldrich) in the absence or presence of the inhibitor of MAPK/ERK kinase U0126 $(20 \mu \mathrm{M})$. After 24 or $48 \mathrm{~h}$ of incubation, CCK-8 reagent (10 $\mu \mathrm{l})$ was added to each well of a 96-well flat-bottomed microplate containing $100 \mu \mathrm{l}$ of culture medium, and the plate was incubated for $1 \mathrm{~h}$ at $37^{\circ} \mathrm{C}$. Viable cells were evaluated by absorbance measurements at $450 \mathrm{~nm}$ using an auto microplate reader. The OD450 value was proportional to the degree of cell survival. All results are representative of three independent experiments.

Western blot analysis. For protein extraction, each sample was made into powder and placed in liquid nitrogen with a precooled mortar and pestle. The tissue powder was constructed using lysis buffer [50 mM Tris-HCl (pH 7.4), $150 \mathrm{mM} \mathrm{NaCl}$, $1 \%$ NP-40, 0.25\% sodium deoxycholate, $1 \mathrm{mmol} / \mathrm{l}$ EDTA,
$1 \mathrm{mmol} / \mathrm{l}$ phenylmethlsulfonyl, $1 \mathrm{mmol} / \mathrm{l} \mathrm{Na}_{3} \mathrm{VO}_{4}$ and $1 \mathrm{mmol} / \mathrm{l}$ $\mathrm{NaF}]$ and incubated at $4^{\circ} \mathrm{C}$ for $30 \mathrm{~min}$, followed by centrifugation at $10,000 \mathrm{x} \mathrm{g}$ at $4^{\circ} \mathrm{C}$ for $20 \mathrm{~min}$. Protein concentrations were then measured using protein assay kits (Bio-Rad). The protein lysates were then resolved by SDS-PAGE and then transferred onto polyvinylidene difluoride membranes (GE Healthcare Biosciences), blocked with PBS containing 0.2\% Tween-20 and 5\% non-fat dry milk and incubated with the primary antibody against BMP6 (1:1,000), P-gp $(1: 1,000)$ and actin $(1: 1,000)$ from Abcam. Antibody binding was revealed by incubation with horseradish peroxidase-conjugated secondary antibodies and the ECL Plus immunoblotting detection system (GE Healthcare Biosciences). Signals were quantified using NIH ImageJ 1.63 software.

Statistical analyses. Data are expressed as the means \pm SD. The paired t-test, one-way ANOVA and Mann Whitney U test and Kruskal-Wallis test were applied for statistical analysis using SPSS software (13.0 version). Statistical significance was set at $\mathrm{P}<0.05$.

\section{Results}

Expression of BMP6 is downregulated and correlates with the clinicopathological parameters in the breast cancer tissues. The RFDD-PCR analyses of mRNA expression profiles between tumor and adjacent tissues identified several gene fragments. These were isolated and subjected to bioinformatic analysis. Among them, BMP6 was identified as being significantly downregulated in the breast cancer tissues (Fig. 1A). Subsequently, real time-PCR and immunohistochemical straining confirmed the expression patterns in the breast cancer and adjacent normal breast tissues. 
Table I. Correlation between BMP6 expression and the clinical characteristics of the breast cancer cases.

\begin{tabular}{|c|c|c|c|c|c|c|}
\hline \multirow[b]{2}{*}{ Characteristics } & \multirow[b]{2}{*}{$\mathrm{n}$} & \multicolumn{4}{|c|}{ BMP6 expression } & \multirow[b]{2}{*}{ P-value } \\
\hline & & - & + & ++ & +++ & \\
\hline Total & 80 & 37 & 14 & 16 & 13 & \\
\hline Age (years) & & & & & & 0.262 \\
\hline$\leq 40$ & 22 & 8 & 6 & 4 & 4 & \\
\hline $41-49$ & 20 & 9 & 5 & 4 & 2 & \\
\hline $50-60$ & 18 & 11 & 2 & 2 & 3 & \\
\hline$>60$ & 20 & 9 & 1 & 6 & 4 & \\
\hline Histology & & & & & & 0.302 \\
\hline Ductal & 34 & 13 & 7 & 8 & 6 & \\
\hline Lobular & 32 & 15 & 6 & 5 & 6 & \\
\hline Other & 14 & 9 & 1 & 3 & 1 & \\
\hline Grade & & & & & & 0.027 \\
\hline 1 & 33 & 14 & 5 & 8 & 6 & \\
\hline 2 & 22 & 10 & 3 & 3 & 6 & \\
\hline 3 & 25 & 13 & 6 & 5 & 1 & \\
\hline ER status & & & & & & 0.030 \\
\hline Positive & 39 & 15 & 6 & 8 & 10 & \\
\hline Negative & 41 & 22 & 8 & 8 & 3 & \\
\hline PR status & & & & & & 0.004 \\
\hline Positive & 36 & 16 & 6 & 6 & 8 & \\
\hline Negative & 44 & 21 & 8 & 10 & 5 & \\
\hline HER2 status & & & & & & 0.282 \\
\hline Positive & 43 & 18 & 10 & 8 & 7 & \\
\hline Negative & 37 & 19 & 4 & 8 & 6 & \\
\hline Ki67 expression & & & & & & 0.012 \\
\hline $0-20 \%$ & 29 & 16 & 3 & 5 & 5 & \\
\hline $21-40 \%$ & 23 & 8 & 5 & 5 & 5 & \\
\hline$>40 \%$ & 28 & 13 & 6 & 6 & 3 & \\
\hline
\end{tabular}

Significant P-values are indicated in bold. BMP6, bone morphogenetic protein 6. ER, estrogen receptor; PR, progesterone receptor; HER2, human epidermal growth factor receptor 2 .

Real-time PCR showed that the BMP6 mRNA expression level was significantly higher in adjacent normal breast tissues when compared to the expression level in the matched breast cancer tissues (Fig. 1B). In tumor tissues, the BMP6 mRNA expression level was significantly higher in estrogen receptor (ER)-positive breast cancers than in ER-negative breast cancers (Fig. 1C). With increasing tumor histologic grade, the expression of BMP6 mRNA was significantly downregulated (Fig. 1D). In addition, we performed immunostaining of 80 breast cancer samples. Cellular staining for BMP6 was detected in both the membrane and the cytoplasm (Fig. 2). Based on the scoring system of immunostaining, the expression of BMP6 was closely related with tumor ER, and progesterone receptor (PR) expression and tumor histologic grade (Table I). Most importantly, the significant association between BMP6 expression and cancer tissue Ki67 status was observed (Table I).

Decrease in BMP-6 expression by shRNA enhances proliferation and chemoresistance in MCF7 cells. Real-time PCR and western blotting revealed that BMP6 mRNA and protein were downregulated in multi-drug-resistant breast cancer cell line MCF7/Adr compared with its parental cell line MCF7 (Fig. 3C). To evaluate the effect of the BMP6 expression in MCF7 cells, the negative control shRNA and BMP6-specific shRNA vectors were transfected into the MCF7 cells and the efficacy of the downregulation of expression of the BMP6 gene was detected by RT-PCR and western blot analysis. The mRNA (Fig. 3A) and protein (Fig. 3B) levels of BMP6 were decreased in the BMP6 shRNA transfection group but not in the control shRNA group.

To evaluate whether silencing the BMP6 gene in MCF7 cells affects cell growth and proliferation, the CCK-8 assay and BrdU labeling analyses were performed. As shown in Fig. 3D, after 5 days of transfection, a significant increase in growth was observed between the NC group cells and the shRNA group cells, respectively. Cell proliferation was also examined by BrdU labeling analysis. The percentage of BrdU-positive cells was significantly lower in the NC group than that in the BMP6 shRNA-transfected cells (Fig. 3E and F). 

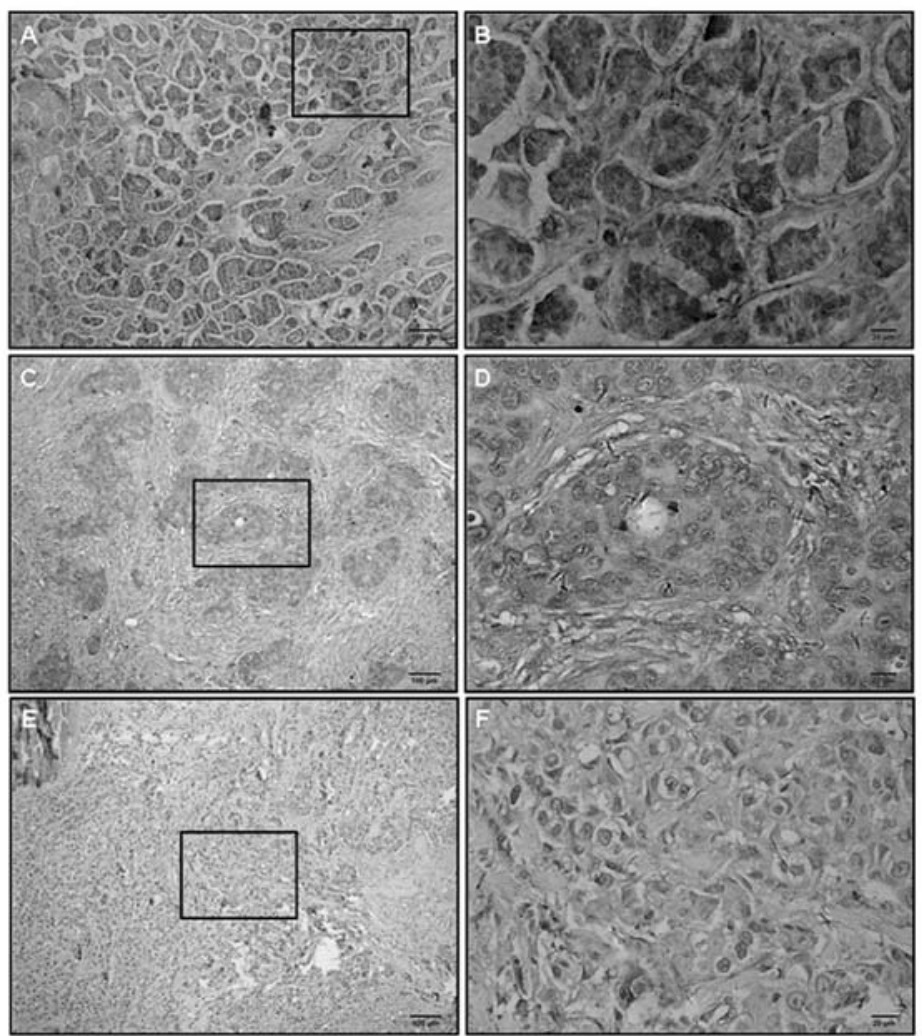

Figure 2. Immunohistochemical analysis of BMP6 expression. BMP6 expression in (A) ER $\alpha$-positive breast cancer tissue and (C) PR-positive breast cancer tissue. (E) Representative image of BMP6 immunostaining in ER $\alpha$-negative breast cancer tissue. B, D and F are high-magnification images of A, C and E, respectively.
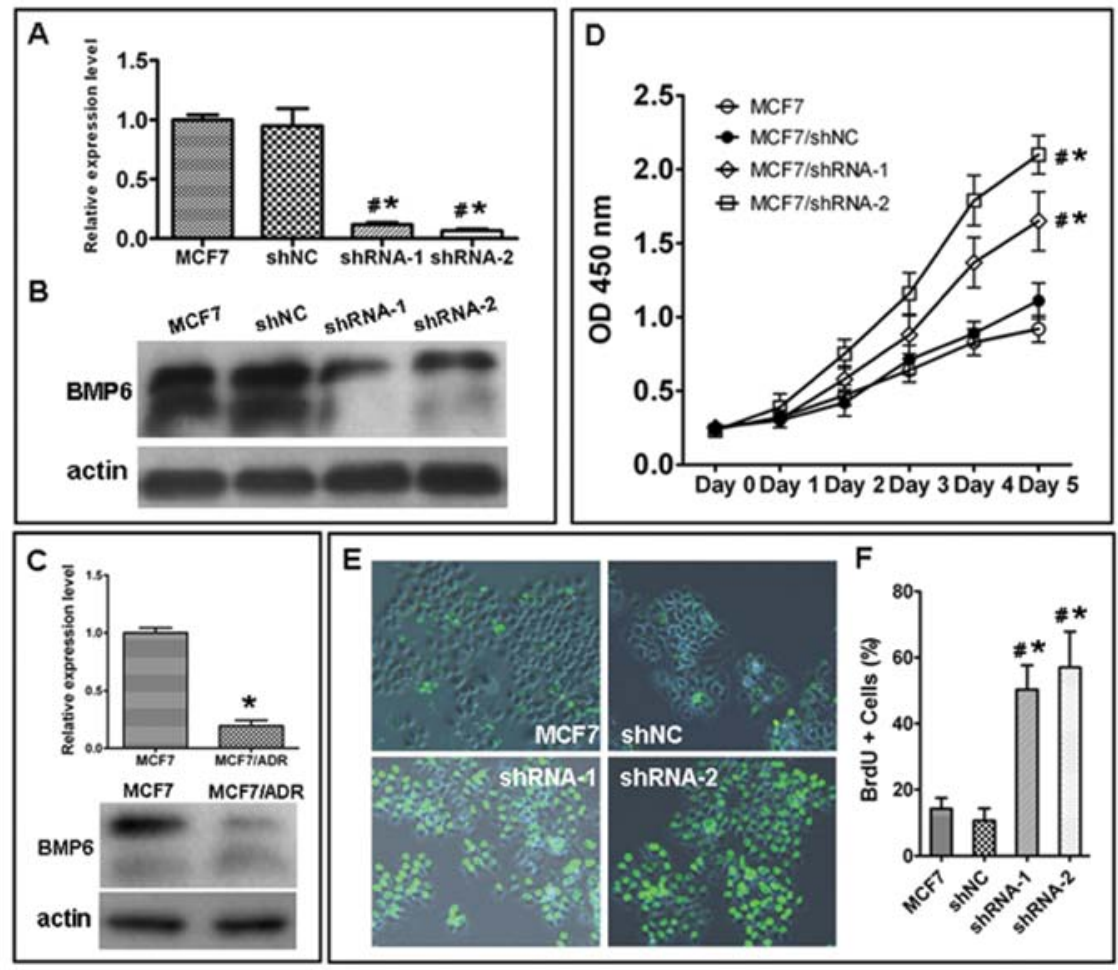

Figure 3. (A) RT-PCR and (B) western blot analysis revealed that transfection of the interference vectors of BMP-6 significantly decreased the BMP-6 expression in MCF-7 breast cancer cells. (C) Real-time PCR and western blot analysis showed that BMP6 mRNA and protein were downregulated in multidrug-resistant breast cancer cell line MCF7/Adr when compared with its parental cell line MCF7. (D) Cell proliferation was determined by CCK-8 assay. At days 1-5, the OD value in each group of cells was measured by absorbance at $450 \mathrm{~nm}$. Treatment of MCF7 cells with BMP-6-specific shRNAs resulted in enhancement of proliferation in a time-dependent manner. (E) Cell proliferation was also confirmed by BrdU labeling (green) in MCF7 cells and cells transfected with shRNA vectors. (F) The percent of BrdU-positive cells in the various groups of cell. All data are shown as means \pm SD of triplicate experiments. ${ }^{*} \mathrm{P}<0.01$ vs. $\mathrm{MCF} 7 ;{ }^{\#} \mathrm{P}<0.01$ vs. $\mathrm{MCF} 7 / \mathrm{shNC}$. 

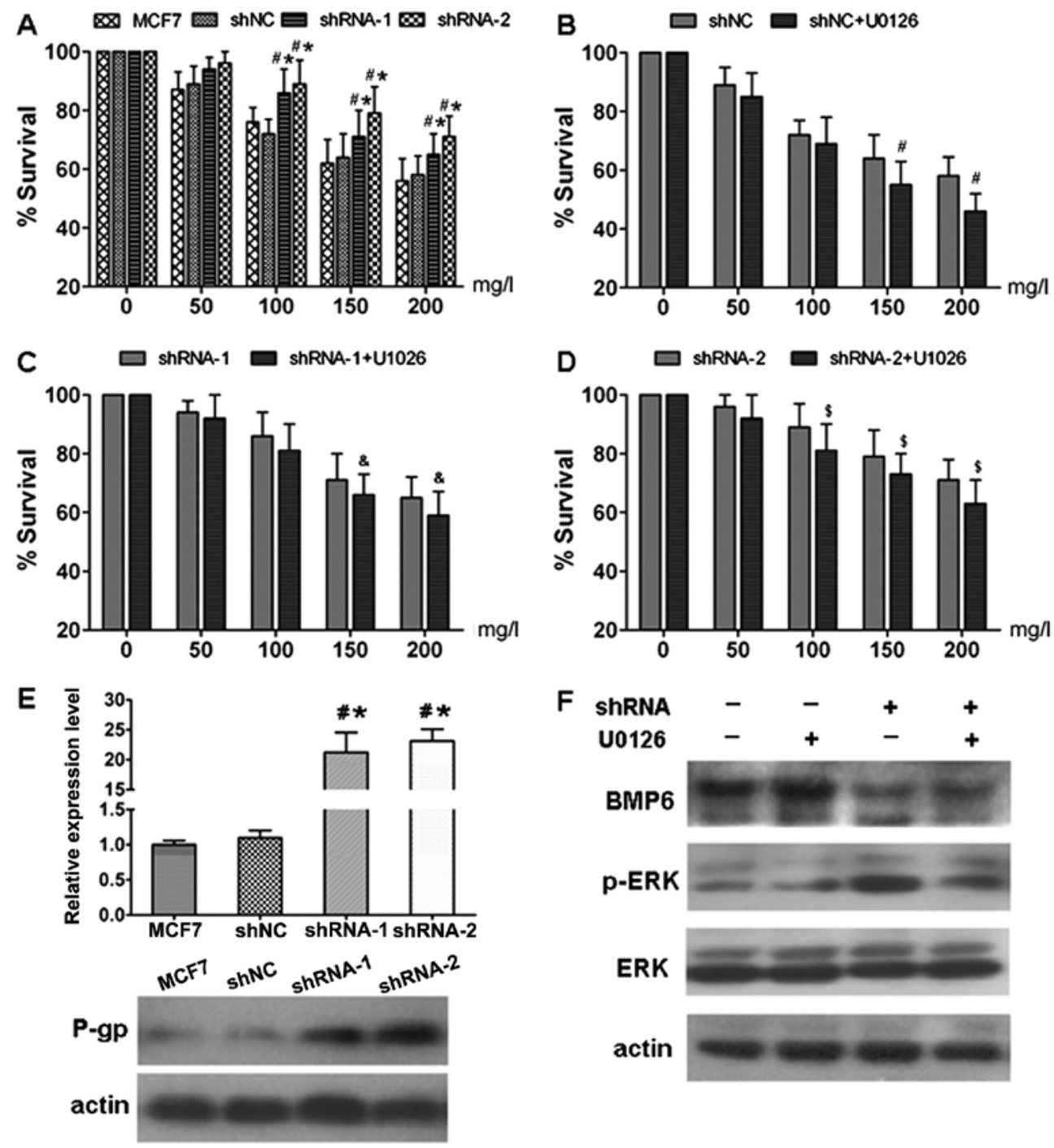

Figure 4. Inhibition of BMP6 expression decreases the chemosensitivity of breast cancer MCF7 cells to doxorubicin. Cell viability was determined by CCK-8 assay. (A-D) MCF7 cells transfected with either the shRNA-BMP6 vectors or control vector were treated with 0, 50, 100, 150 and $200 \mathrm{mg} / 1$ doxorubicin or in combination with $10 \mu \mathrm{M} \mathrm{U} 0126$ for $24 \mathrm{~h}$. The percentage of viable cells in the cells treated with the different concentrations of agents was normalized to that of the untreated controls. All experiments were performed at least in triplicate ( ${ }^{*} \mathrm{P}<0.01 \mathrm{vs.} \mathrm{MCF7;}{ }^{*} \mathrm{P}<0.01 \mathrm{vs}$. MCF7/shNC; ${ }^{\&} \mathrm{P}<0.01 \mathrm{vs}$. MCF7/shRNA-1; ${ }^{\mathrm{s}} \mathrm{P}<0.01 \mathrm{vs}$. MCF7/shRNA-2). (E) Western blot analysis showed that the MDR1 gene mRNA or protein expression was upregulated significantly in the MCF7 cells transfected with the shRNA-BMP6 vectors. (F) Western blot analysis of BMP-6 and p-ERK levels after treatment with the BMP6-specific shRNA vectors in the absence or presence of the MAPK inhibitor U0126.

Furthermore, the effects of BMP6 expression on doxorubicin resistance in the BMP6-specific shRNA transfected and parental cells were assessed by growth inhibition analysis using the CCK-8 assay. The survival ratio of MCF7, MCF7/shNC, MCF7/shRNA-1 and MCF7/shRNA-2 cells was analyzed after treatment with a range of increasing concentrations of doxorubicin for $24 \mathrm{~h}$ by CCK-8 assay. As shown in Fig. 4A, following treatment with doxorubicin for $24 \mathrm{~h}$, number of surviving MCF7/shRNA-1 and MCF-7/shRNA-2 cells was significantly increased compared to the number of cells in the MCF7 and MCF7/shNC cells. There was no significant difference in doxorubicin resistance between the MCF7 and MCF7/shNC cells. These results suggest that inhibition of BMP6 expression in MCF7 cells decreased the sensitivity to the cytotoxic effects of doxorubicin.

BMP6 knockdown increases $m d r-1 / P$-gp expression and activates the ERK signaling pathway. As shown in Fig. 4E, expression of the multi-drug resistance gene mdr-1 and protein P-gp were significantly downregulated after BMP6 knockdown in the MCF7 cell lines. Furthermore, the activation of the ERK signaling pathway was observed in MCF7 cells transfected with BMP6-specific shRNA. The increased level of p-ERK was attenuated by the MAPK/ERK inhibitor U0126 (Fig. 4F). In addition, U0126 also reduced the survival of MCF7 cells transfected with the BMP6-specific shRNA following treatment with doxorubicin (Fig. 4B-D). These results indicate that enhancement of MCF7 cell chemoresistance induced by BMP6 knockdown involves ERK signaling pathway activation.

\section{Discussion}

BMPs have been found to be associated with various types of cancers such as breast cancer, prostate cancer, multiple myeloma and ovarian cancer (6). Recently studies of various 
cancer models have indicated the critical role of the BMP signaling pathway in tumor cell carcinogenesis, through autocrine and paracrine mechanisms, resulting in the modulation of tumor cell growth, invasion and metastasis (12-14). Regarding BMP6, previous studies have shown that it is expressed in primary breast cancer samples and cell lines and functions as an inhibitor of breast cancer cell growth and migration (7). However, the relationship between BMP6 expression and histopathological characteristics of breast cancer and the function of BMP6 in breast cancer chemoresistance are yet unclear. In the present study, we showed that BMP6 expression was significantly downregulated in the majority of primary breast cancer specimens when compared with that in the adjacent normal tissues by RFDD-PCR, real-time PCR and immunohistochemistry. In the tumor tissues, the BMP6 mRNA or protein expression was significantly correlated with breast cancer tumor grade and ER and PR statuses. BMP6 expression in ER-negative breast cancer was obviously lower than that in ER-positive breast cancer. Previous studies have shown that promoter hypermethylation contributes to the downregulation of BMP6 expression in ER-negative breast cancer patients $(15,16)$. Our results were in agreement with a previous observation (7) and moreover, further analysis also revealed a significant association between tumor cell grade and BMP6 expression in tumor tissues. More importantly, we found that the expression of BMP6 was closely related to the Ki67 expression of tumor cells, which is recognized as a marker for tumor cell proliferation. In order to confirm the function of BMP6 in breast cancer cell proliferation, an shRNA expression vector targeting the BMP6 gene was constructed and transfected into MCF7 cells. The results showed that inhibition of the expression of BMP6 significantly enhanced cell proliferation. Notably, BMP6 was found to inhibit cell proliferation in various cancer types including breast cancer, multiple myeloma, prostate cancer and skin tumors via multiple mechanisms including suppression of the p38-MAPK signaling pathway, cell cycle arrest and downregulation of AP-1 expression (17-20). These data suggest that BMP6 may be a new diagnostic marker of breast cancer, and deregulation of BMP6 is involved in loss of proliferation control and failure to undergo cellular differentiation during tumorigenesis.

Cancer cell chemoresistance is a major obstacle to cancer treatment. Chemoresistance accounts for most of the failure in adjuvant chemotherapy in breast cancer. To date, several mechanisms have been proven to play an important role in the chemoresistance phenotype (21-23). In the present study, we first noted that BMP6 expression was associated with drug resistance. Previous studies have shown that BMPs are involved in metastasis, epithelial to mesenchymal transformation, altered cellular behavior and angiogenesis in human cancer, and are closely related to drug resistance (24-26). In addition, we also found that BMP6 mRNA and protein were downregulated in the multi-drug-resistant cell line MCF7/ADR. Therefore, we hypothesized that BMP6 expression is involved in breast cancer drug resistance. In the present study, BMP6 knockdown by BMP6-specific shRNAs in MCF7 cells resulted in a significant upregulation of P-glycoprotein, one of the well characterized factors of breast cancer multidrug resistance encoded by the mdr-1 gene. As known, BMPs regulate the gene expression through the smad-dependent and smad-independent signaling pathway $(27,28)$. Here, we showed that BMP6 knockdown activated the expression of the ERK signaling pathway and elevated the resistance of MCF7 cells to the chemotherapy drug doxorubicin. Moreover, the selective MAPK/ERK signaling pathway inhibitor U0126 attenuated the chemoresistance of MCF7 cells resulting from BMP6 knockdown. In fact, our previous and other various studies have also confirmed that ERK signaling pathway activation is involved in cancer cell chemoresistance $(23,29)$. ERK signaling pathway-modulated mdr-1 gene expression was also confirmed in various studies $(30,31)$. Thus, these findings suggest that downregulation of BMP6 upregulates mdr-1/p-gp expression by activating the ERK signaling pathway. Further analysis of the related mechanisms is needed to fully assess the function of BMP6 in breast cancer cell chemoresistance.

In summary, we demonstrated the expression pattern of BMP6 in breast cancer and revealed that the downregulation of BMP6 was associated with breast cancer grade, enhanced cancer cell proliferation and chemoresistance. Furthermore, BMP6 knockdown enhanced breast cancer cell chemoresistance via activation of the ERK signaling pathway. Our results provide evidence suggesting a critical role of BMP6 in breast cancer, and BMP6 may serve as a novel diagnostic biomarker or therapeutic target for breast cancer.

\section{References}

1. Cancer Genome Atlas Network: Comprehensive molecular portraits of human breast tumours. Nature 490: 61-70, 2012.

2. Gao H, Chakraborty G, Lee-Lim AP, et al: The BMP inhibitor Coco reactivates breast cancer cells at lung metastatic sites. Cell 150: 764-779, 2012.

3. Cellurale C, Girnius N, Jiang F, et al: Role of JNK in mammary gland development and breast cancer. Cancer Res 72: 472-481, 2012.

4. Perrimon N, Pitsouli C and Shilo BZ: Signaling mechanisms controlling cell fate and embryonic patterning. Cold Spring Harb Perspect Biol 4: a005975, 2012.

5. Guo $\mathrm{J}$ and $\mathrm{Wu} \mathrm{G}$ : The signaling and functions of heterodimeric bone morphogenetic proteins. Cytokine Growth Factor Rev 23: 61-67, 2012

6. Singh A and Morris RJ: The Yin and Yang of bone morphogenetic proteins in cancer. Cytokine Growth Factor Rev 21: 299-313, 2010.

7. Alarmo EL and Kallioniemi A: Bone morphogenetic proteins in breast cancer: dual role in tumourigenesis? Endocr Relat Cancer 17: R123-R139, 2010.

8. Rhodes A, Jasani B, Barnes DM, et al: Reliability of immunohistochemical demonstration of oestrogen receptors in routine practice: interlaboratory variance in the sensitivity of detection and evaluation of scoring systems. J Clin Pathol 53: 125-130, 2000.

9. Livak KJ and Schmittgen TD: Analysis of relative gene expression data using real-time quantitative PCR and the 2(-Delta Delta C(T)) method. Methods 25: 402-408, 2001.

10. Deng S, Zhou H, Xiong R, et al: Over-expression of genes and proteins of ubiquitin specific peptidases (USPs) and proteasome subunits (PSs) in breast cancer tissue observed by the methods of RFDD-PCR and proteomics. Breast Cancer Res Treat 104: 21-30, 2007.

11. Zhou HY, Mei Y, Lu YG, et al: Application of restriction fragment differential display-polymerase chain reaction in study on differential expression profiles of human diseases. Zhonghua Yi Xue Yi Chuan Xue Za Zhi 22: 294-297, 2005.

12. Piccirillo SG and Vescovi AL: Bone morphogenetic proteins regulate tumorigenicity in human glioblastoma stem cells. Ernst Schering Found Symp Proc 5: 59-81, 2006.

13. Bunyaratavej P, Hullinger TG and Somerman MJ: Bone morphogenetic proteins secreted by breast cancer cells upregulate bone sialoprotein expression in preosteoblast cells. Exp Cell Res 260: 324-333, 2000. 
14. Shepherd TG, Thériault BL and Nachtigal MW: Autocrine BMP4 signalling regulates ID3 proto-oncogene expression in human ovarian cancer cells. Gene 414: 95-105, 2008.

15. Barekati Z, Radpour R, Lu Q, et al: Methylation signature of lymph node metastases in breast cancer patients. BMC Cancer 12: $244,2012$.

16. Zhang M, Wang Q, Yuan W, et al: Epigenetic regulation of bone morphogenetic protein- 6 gene expression in breast cancer cells. J Steroid Biochem Mol Biol 105: 91-97, 2007.

17. Seckinger A, Meissner T, Moreaux J, Goldschmidt H, Fuhler GM Benner A, et al: Bone morphogenic protein 6: a member of a novel class of prognostic factors expressed by normal and malignant plasma cells inhibiting proliferation and angiogenesis. Oncogene 28: 3866-3879, 2009.

18. Takahashi M, Otsuka F, Miyoshi T, et al: Bone morphogenetic protein 6 (BMP6) and BMP7 inhibit estrogen-induced proliferation of breast cancer cells by suppressing p 38 mitogenactivated protein kinase activation. J Endocrinol 199: 445-455, 2008.

19. Haudenschild DR, Palmer SM, Moseley TA, You Z and Reddi AH: Bone morphogenetic protein (BMP)-6 signaling and BMP antagonist noggin in prostate cancer. Cancer Res 64: 8276-8284, 2004.

20. Wach S, Schirmacher P, Protschka M and Blessing M: Overexpression of bone morphogenetic protein-6 (BMP-6) in murine epidermis suppresses skin tumor formation by induction of apoptosis and downregulation of fos/jun family members. Oncogene 20: 7761-7769, 2001.

21. Coley HM: Mechanisms and consequences of chemotherapy resistance in breast cancer. Eur J Cancer (Suppl 7): 3-7, 2009

22. Drasin DJ, Robin TP and Ford HL: Breast cancer epithelial-tomesenchymal transition: examining the functional consequences of plasticity. Breast Cancer Res 13: 226, 2011.
23. Chen GQ, Zhao ZW, Zhou HY, Liu YJ and Yang HJ: Systematic analysis of microRNA involved in resistance of the MCF-7 human breast cancer cell to doxorubicin. Med Oncol 27: 406-415, 2010.

24. Yang S, Du J, Wang Z, et al: Dual mechanism of deltaEF1 expression regulated by bone morphogenetic protein- 6 in breast cancer. Int J Biochem Cell Biol 41: 853-861, 2009.

25. Yang S, Du J, Wang Z, et al: BMP-6 promotes E-cadherin expression through repressing deltaEF1 in breast cancer cells. BMC Cancer 7: 211, 2007.

26. Foroni C, Broggini M, Generali D and Damia G: Epithelialmesenchymal transition and breast cancer: role, molecular mechanisms and clinical impact. Cancer Treat Rev 38: 689-697, 2012.

27. Zhang YE: Non-Smad pathways in TGF-beta signaling. Cell Res 19: 128-139, 2009.

28. Li B: Bone morphogenetic protein-Smad pathway as drug targets for osteoporosis and cancer therapy. Endocr Metab Immune Disord Drug Targets 8: 208-219, 2008.

29. Karam M, Legay C, Auclair C and Ricort JM: Protein kinase D1 stimulates proliferation and enhances tumorigenesis of MCF-7 human breast cancer cells through a MEK/ERK-dependent signaling pathway. Exp Cell Res 318: 558-569, 2012.

30. Shen $\mathrm{H}, \mathrm{Xu} \mathrm{W}$, Luo W, et al: Upregulation of mdr1 gene is related to activation of the MAPK/ERK signal transduction pathway and YB-1 nuclear translocation in B-cell lymphoma. Exp Hematol 39: 558-569, 2011.

31. Guan J, Chen XP, Zhu H, Luo SF, Cao B and Ding L: Involvement of extracellular signal-regulated kinase/mitogen-activated protein kinase pathway in multidrug resistance induced by $\mathrm{HBx}$ in hepatoma cell line. World J Gastroenterol 10: 3522-3527, 2004. 